\title{
Effectiveness of 3D Scanning in Establishing Sideseam Placement for Pattern Design
}

\author{
Kathryn BROWNBRIDGE*a , Simeon GILL $^{\mathrm{a}}$, Susan ASHDOWN ${ }^{\mathrm{b}}$ \\ a Manchester Metropolitan University, Manchester, UK; \\ ${ }^{\mathrm{b}}$ Cornell University, Ithaca NY, USA
}

http://dx.doi.org/10.15221/13.041

\begin{abstract}
As woven garments are cut from flat pieces of cloth, pattern cutting methods must create flat 2D shapes that are constructed to form 3D garments that fit a particular body shape. The placement of sideseams represents a key division of the pattern where the front and back portions of a garment relating to a particular body measurement are distributed to create a balanced garment. During most pattern creation methods the distribution of the measurement into back and front arcs is proportionally determined by the author, however body scanning provides new opportunities to understand the body and derive these arcs during the measurement process. TC2 measurement software provides the facility to automatically derive arcs based on non-disclosed divisions of the body along a vertical plane. This research tests the automated division of key circumferences into arcs using the TC2 software, against methods guided by practitioner experience and placed using non-automated 3D software. Focusing on circumferences of the bust, waist and hip, analysis of the arcs derived by the scanner from a number of standard female body forms and 10 scans of female subjects, will be tested against those determined by a panel of experts. These methods form the basis for an understanding of how circumference division can be automated and allow for the testing of these methods on a variety of different scans and the comparison of the arcs against those proposed or applied in standard industry practice. It will be possible to see how closely these methods match or contrast with these imposed systems. This exploration provides a clear link to body pattern relationships and provides a foundation from which to advance mass customization utilizing body scanning technology and automated arc definition.
\end{abstract}

Keywords: $3 d$ body scanning, Anthropometrics, Pattern Construction

\section{Introduction}

The automation of pattern construction driven by anthropometric analysis of the human body has, with recent developments in body scan capture and software programs, become a key area of focus for future garment development. Construction of the block, the basic unstyled pattern development tool, is often achieved using a proprietary drafting system which instructs on the creation of a shape based on body measurement, proportional rules and the inclusion of ease [1]. These systems are nuanced and require some familiarity on behalf of the practitioner to apply successfully. They are historically based on common practice, as data on anthropometric measures from the population were not available to guide the development of the original systems. Further to this techniques and tools applied in modern pattern construction such as standard units of measure are examples of concurrent development which makes evolving theories difficult [2]. Therefore current pattern construction methods exhibit a fair amount of variation, as different practitioners develop independent theories based on their own experiences and fashion influences of the time. As patterns are accepted to relate directly to the body and historically have been developed through draping as well as drafting [2-4], there is growing recognition that it should be possible to create these shapes directly from the body surface within virtual environments [5,6]. More recently methods have been proposed that provide more technologically developed solutions $[7,8]$ though these still leave questions regarding pattern theory. This lack of theory highlights the striking number of assumptions that practitioners must make when generating patterns and provide the basis for discussion of the issues that need to be resolved in order to make effective automation of pattern shapes possible.

Pattern drafting systems can provide the basis for development of automated systems, but they employ assumptions that must be reduced to a provable theory to develop valid and reliable results. However, as pattern construction is historically a practice based development, there is little by way of consistent theory on which to relate practice. The importance of the sideseam, and inclusion of a method of identifying and patterning to the sideseam is one such theoretical issue, which has a strong bearing on developing automated pattern construction systems. Armstrong [9] is one of the few authors whose pattern methods require arc measurements to suitably define the back and front portions of a typical pattern. Other methods, including Aldrich [10], Bunka Fashion [11] and Beazley \& 
Bond [12] all recommend the collection of whole circumferences, which are then divided into the commonly used front and back pattern pieces according to undisclosed proportions, during the pattern construction process. The proportions considered to be appropriate within many of the major pattern methods for creating the bodice for a standard UK size 12 dress form vary from $49-58 \%$ for the front waist arc and $50-55 \%$ for the front bust arc [13]. This indicates a variation in proportional techniques for dividing the arcs that would make it difficult to automate this process from purely documented practice based research focused on existing methods of pattern construction.

Without explicit development of theory regarding patterns and proportions, it is difficult to create a valid and reliable process for automating the process of pattern creation. This paper addresses some of the difficulties of establishing arc divisions from scan data, based on automated measurement placement and addresses one of the key required theoretical underpinnings to enable suitable pattern automation in developing systems. The focus will be predominantly on sideseam placement relative to the arc divisions of the bust, waist and hip that represent key dimensions in the construction of garment patterns.

\section{Current understanding of patterns and sideseam placement}

Tao and Bruniaux [7] provide a very current example of developments in the creation of garments using a combination of $3 \mathrm{D}$ and manual techniques. By moving between $2 \mathrm{D}$ and $3 \mathrm{D}$ systems, they propose methods for automated development of trouser patterns using virtual avatars. Whilst the method shows how person/pattern relationships can be controlled, the reliance on a single 2D pattern construction method to support their development does not address fundamental areas of practice in pattern construction. The study in using only a single pattern construction system to guide development and determine pattern divisions and proportions removed the difficulties of individual practices imposed by varied methods [1]. Decisions such as suppression, side seam placement and proportions of the pattern dictated by the learned practice in the method applied avoid difficulties of other documented methods and the lack of definable theory that can communicate common aspects of pattern construction.

\subsection{Defining the sideseam placement}

The developing technologies of the late 90's that made it possible to capture and create the human body in virtual environments supported developments in automated pattern generation. Kang and Kim [14] provide an early example of how the 3D body surface can be used to create 2D pattern shapes with a direct relationship to the body surface. However, this method does not address either the addition of ease or the fundamental identification of sideseam placement.

Analysis of major western pattern cutting methods for bodices conducted by Gill [13] establishes that most bodice patterns apply proscribed divisions to whole circumferences to create the front and back arcs that define current patterns. However, this work does not document the variation in proportions defined in the construction method relative to the proportions of the individual figure in a manner that would define what happens relative to different proportions. An exception to current methods of using whole circumferences is Armstrong [9] who defines arcs in the measurement process, though these measurements are collected from the body form and no clear details of sideseam location on individual bodies is provided [13]. The manufacturer relying on common practice determines the placement of the sideseams on the dress forms; this placement can be arbitrary. Manufacturing variation can also affect the placement of sideseams of the dress forms as their creation is essentially a manual process, and the reliability of the placement is dependent on the skill of the technician. Recent manufacturing processes in the industry have introduced better quality control and more reliable results (Alvanon.com), though as there is no universal definition of sideseam divisions on the body there is little to guide their determination of this position on the dress form.

Most apparel practitioners would agree that the sideseam should be vertical, i.e. perpendicular to the ground $[15,16]$. Many use some part of the armscye as a landmark. For example, Farmer and Gotwals [15] identify the center of the armscye as the appropriate landmark. Both Hazen [17] and Rasband [16], say that the sideseam should extend from the center of the underarm. Other methods concentrate on body proportions. Liechty et al.[18], claim the sideseam should "divide the body in becoming proportions." and the instructions in Vogue Fitting, say that the sideseam should be "centered on the body" from the side view [19]. Liechty et al. [18] also says that the sideseam should appear to intersect the waistline at a 90 angle, and that it should be placed so that it creates an equal visual distance from the front, side, and back, i.e. the sideseams should not be visible from the front or back view of the body and should be centered on the side view of the body. 
In a study from 2007 of sideseam placement seven apparel practitioners with at least ten years of experience were asked how they would place a sideseam [20]. Six of the seven judges indicated that they used at least two methods. The following methods were described:

(1) centering on the profile at a body landmark (under the arm, at the waist, at the hip);

(2) generating a vertical line from a landmark (the ear lobe, the shoulder point, the position of fingers

in relaxed posture at side, the center of the armscye, the hip joint); or

(3) looking for balance among front and back points of greatest protrusion

(shoulder blade to bust, bust to hip, shoulder to hip, and leg position).

Given the variety of responses, it is clear that there is no generally accepted (correct) placement of the sideseam. As apparel construct, it has been defined by professional practice, with individual preferences in methods of selection. In order to effectively automate this process it is necessary to agree on an appropriate scan measurement procedure.

\subsection{Function of sideseams in pattern construction}

Sideseams are not universally used in apparel (tubular knits do not have vertical sideseams, and for some tailored garments a side panel displaces the seam to the side front and side back[4, 21]); however the use of sideseams are fairly ubiquitous. The use of a seam at the side of the body limits the width of panels of the garment and limits the distance that woven fabric panels need to wrap around the body. This has advantages in controlling grain of the fabric, and results in panel sizes that can be cut efficiently saving fabric in the layout (marker). However, an improperly placed sideseam can result in an imbalance in the hang of the garment, or in an awkward visual division of the body [22]. The importance of line is one of the fundamental aspects of creating well fitted garments, which are accepted as a staple in much of the existing clothing research. The five principals of fit, grain, line, set, balance and ease set out by Erwin, Kinchen and Peters [22] provide a sound foundation on which to define fit, primarily for tailored garments and suggest the consideration of the garment when defining how the dimensions of the body are used in its development. The importance of balance during pattern construction is mentioned in the Bunka guides relative to waist location [11], but is rarely given consideration in many of the existing guides at the stage of pattern construction. Bunka suggest the importance of practitioner judgment to impose a balance subject to the practitioners preferences which will also be influenced by the time period of the construction methods development [1].

The body is considered largely symmetrical across the sagittal plane, as the right and left side of the body are essentially considered as mirror images for most healthy people. This is also borne out by current pattern practice where patterns are developed as symmetrical halves[1]. However, the same is not true for the body divided on the frontal plane. The front half of the body is shaped entirely differently than the back of the body. The balance of the body from front to back is also an area of great proportional variation in the population. This proportional variation is not captured in simple circumferential measures. For example, two women with the same bust circumference measurement may have a different distribution of body shape; one woman may have a flat back and full bust, while another has a flatter bust and well-developed shoulders and back. Arc measurements from sideseam to sideseam would be different for these two women, and the widths of front and back panels at the bust would vary in well-fitted, balanced patterns developed for them. Current practice in pattern construction dictate that alterations for individual morphologies of this nature are made during the pattern testing and fitting process $[9,10,18]$, rather than at the stage of pattern development, precluding automated pattern development systems.

Sideseam placement on individual bodies is not a simple process. No clear body landmarks exist that can reliably capture the sideseam placement that will result in a balanced garment. Unlike most body measurements, the arc measurement is based on an apparel construct that has no 'natural' placement related to body configuration. This lack of a clear body landmark is evident when one investigates sideseam placement definitions in the literature. Though apparel practitioners regularly make the decision of sideseam placement there are a variety of theories on how this should be done.

\subsection{Future development}

Current measurement definitions for scanning are grounded in the protocols of ergonomics [23] and pay little attention to the application of measurements in clothing. The requirements to engage with measurement application ensures that practitioner knowledge must play a role in how we look to automate pattern construction and the determination of suitable divisions that will provide data that can drive the many means of pattern development. Until existing methods of automated pattern 
generation [7, 14] engage with variation in pattern practices [1] there will remain a disconnect between data collected from the body and its application in the development of clothing.

\section{Methodology}

This work used body scanning technology to capture data from dress forms and from real bodies to better understand existing methods for determining the placement of sideseams related to different bodies and theories. Further context was provided with reference to studies of pattern construction method.

\subsection{Comparison of practitioners sideseam location}

Body scans were captured using either a TC2 KX16 or a Human Solutions Vitus XXL scanner, scans in their raw format were taken into Geomagic and converted to similar file types to produce tiff images from which sideseam placement could be visually judged by a panel of seven experienced apparel industry practitioners. The seven practitioners had an average of 30 years' experience of working in the clothing industry in varied roles, the least experienced having 14 years and the most 43 years. Side seams were marked on side profiles of the 24 scan images, including the seven primarily used in this study, using Adobe illustrator. The ruler function was used to ensure sideseams were vertically aligned and sideseams were marked with an orange line as this showed most effectively on the blue profiles. Relative depths of sideseams were compared between practitioners in relation to the distance the division would occur from the front most point of each scan in the measurement lines of bust, waist and hip.

\subsection{Comparison of TC2 automated sideseam location}

All scans were then processed into a BIN format which could be opened in TC2 scan software and allowed body models to be created which could be interrogated using the automated measurement functions. A custom MEP (measurement extraction parameters) was used to extract circumferences and arcs from the bust, waist and hip, the definitions selected accord well with measurement definitions used in recent major sizing surveys, SizeUK and SizeUSA. These measurement arcs and their correspondent landmarks suggested divisions of the body, which would provide the markers for automated sideseam placement used in any future application of the data to drive automated pattern construction. The side on visual of the scan with the arc lines marked was captured as a screenshot and using Corel Photo-paint X5 converted to a Jpeg image. Vertical lines were marked on each scan image aligned with the right side waist point, to indicate the orientation of sideseam division and provide a means to visually determine the proposed orientation against the bust and hip divisions.

\subsection{Comparison of measurement divisions from TC2 software}

Arc measurements alongside full circumferences were output from each of the scans to determine the amount of the circumference considered to be within the front and back arc portions for each of the different scans. These results show relative lengths as portions of the circumference rather than as depths through the form.

\subsection{Comparison of arcs by pattern practice}

Utilizing existing data of measurements determined from analysis major methods of bodice pattern creation [13] further analysis was undertaken to establish arc divisions within the pattern at the bust and waist as a percentage of the overall pattern circumferences at these dimensions.

\section{Results and Discussion}

The following sections outline the findings from the individual aspects of the study and provide details of how these inform considerations for future development in terms of practice and technology.

\subsection{Comparison of practitioner's sideseam location}

Seven different practitioners determined sideseam locations on the Seven dress forms used within this study (Figure 1) as well as a further 17 images based on a combination of dress forms and scans. The following discussion deals with the results of all images. 


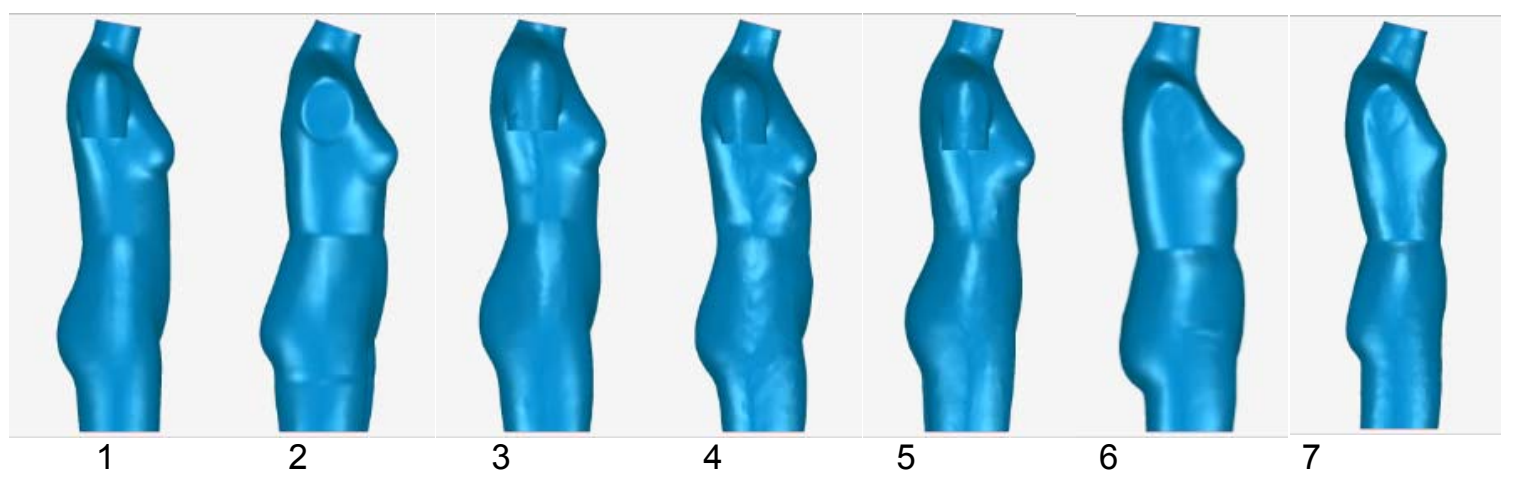

Figure 1: Scan images used in sideseam placement. 1.AsianAlva; 2.DressRite_size8; 3.MMU-WMS012C-0807; 4.MMU-WMS012S-0807; 5.WMS0006-0507; 6.Wolf_m1959_size14; 7. Wolf1997_size3]

Placement of the sideseam by the panel of industry practitioners showed a range of variation in conceptual basis for these decisions. Based on a comparison of depth values from front landmarks to the sideseam placement, practitioner \#5 consistently chose a position that was closer to the posterior of the form or body than the other members of the panel, choosing the position furthest to the rear in 14 of the 28 cases. Practitioner \#2 also favored a more posterior placement, and showed the next highest average value of front landmark to sideseam. On the other hand, practitioner \# 6 chose the most anterior position (12 of the 28 cases), and practitioner \# 7 had the next lowest average value of front landmark to sideseam, identifying a relatively forward sideseam position (see Figure 2). Practitioner \#4 showed the most internal variation in placement, placing the sideseam in the most posterior position of all the practitioners in three cases and in the most anterior position in three cases.

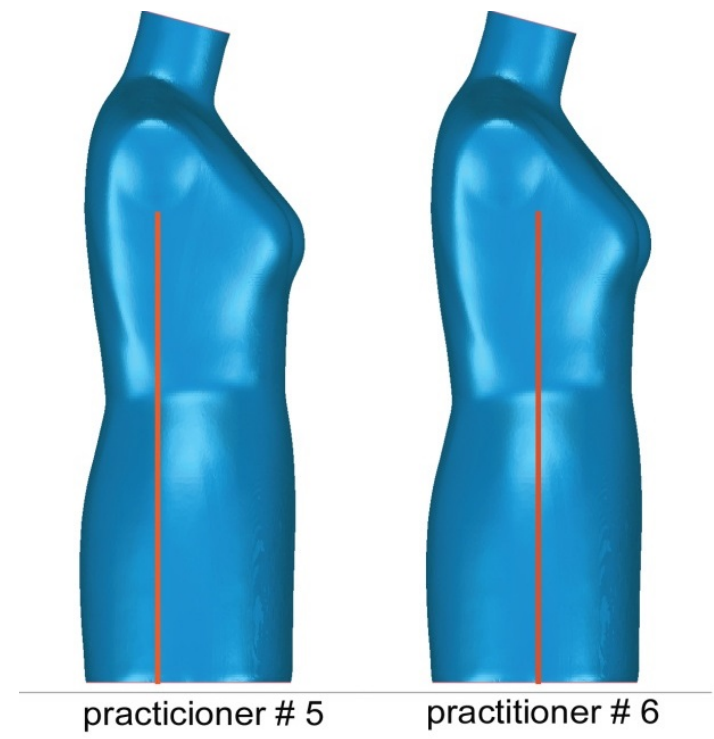

Figure 2: Variation in sideseam placement by apparel practitioners.

The variation within a single practitioner's selection of sideseam positions is not unexpected. The sample bodies that were selected included a range of torso types, from very small to very large, and from standardized dress forms to actual bodies that exhibited a wide range of proportional and postural variation could result in different best practice placement to accommodate various patternmaking and aesthetic decisions.

Various decisions on sideseam placement will result in different patternmaking outcomes, with the compromises inherent in any decision. A comparison of the contrasting decisions in Figure 2 can illustrate some of these compromises. The more posterior position on the left results in a sideseam that is suspended in the center of the armscye, and that hangs directly below, and creates a visual continuity from the highest point of the shoulder. On the other hand, the side torso is unevenly divided, and the fabric in the front panel would need to be much wider than the back. If there are devices for shaping the fabric to the body (such as darts or dart equivalents) that cross the sideseam then the device is extended through a wider surface, and is further from the shaped portion of the 
body that is being addressed. The position on the left centers the sideseam in the pane of the side view, balances the front and back panel sizes, and keeps any control points on the sideseam closer to the more shaped portions of the body. The opportunity for variation in practitioner placement also raises the issue of how automated functions would accommodate required changes by the practitioner and account for the considerations of fabric and suppression.

\subsection{Sideseam division from the TC2 software}

Using the measurement extraction parameters from TC2 software key dimensions were determined on seven of the dress forms. The following images show how sideseam divisions would occur using the arcs in relation to a vertical line from the side waist points.

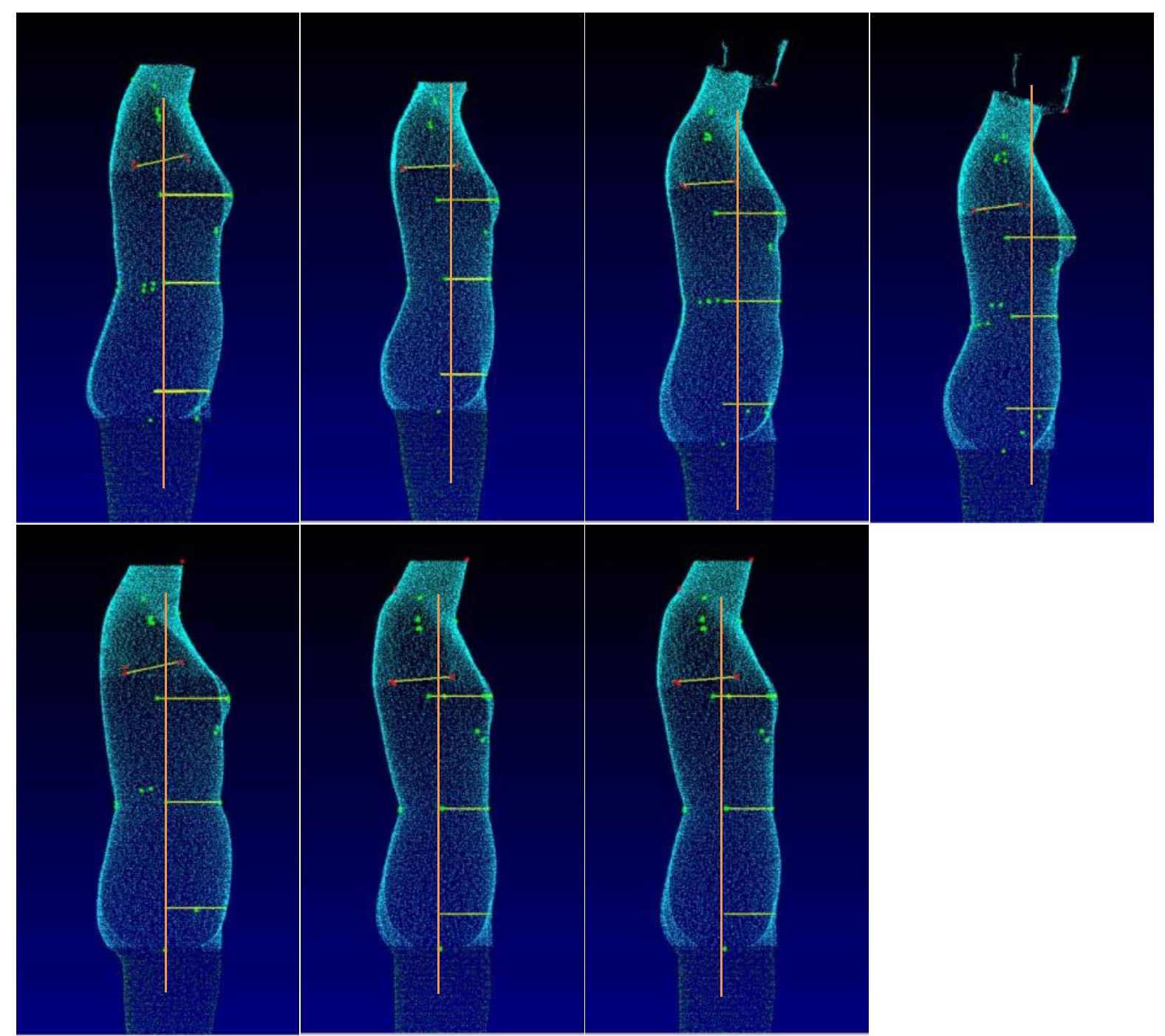

Figure 2: Images of sideseam placement on the dress form scans [scans from left to right: 1.AsianAlva; 2.DressRite_size8; 3.MMU-WMS012C-0807; 4.MMU-WMS012S-0807; 5.WMS0006-0507; 6.Wolf_m1959_size14; 7. Wolf1997_size3]

Each of the dress forms shows a different posture and when sideseam division is vertically placed, it does not align with the divisions of the body at the bust and hip. There is also a different balance to the centre of the armhole area on the scans as determined by the depth of the armscye relative to the front and back armpit points. The analysis of scan data suggests the derivation of sideseams based on armhole centers relative to the armscye width will not be appropriate. Variation even within dress forms size and shape suggest current arc divisions hardwired in to TC2 software will not create sideseams, which are vertical to the body. Most arc point's pitch the waist side points slightly forward of the line created through the bust and in some cases the hip. Consideration should be given to defining the sideseam vertically using coordinates that enable suitable determination of arcs relative to their application. 
Interrogation of the TC2 software and the MEP definitions shows that waist divisions can be altered to break the arc toward the back or front of the body but not place it relative to the planes dividing the bust and hip arcs. Further consideration is required to the related nature of body points used to define and guide pattern construction. Analysis of the sideseam definition within the TC2 MEP editor indicates that the upper body sideseam division is set as a plane beginning at the waist division and running vertically in the coronal plane at the average depth through the body of the two shoulder points. This front to back division will therefore be influenced by the waist size and position of the shoulder relative to the scan subject posture. Similarly the lower arc division references the same side waist points, but is set in the coronal plane according to the crotch point. This means of dividing the body above and below the waist will not always return an arc value that consistently with the three key measurements of bust waist and hip will define a vertical split suitable for defining sideseam for garments covering the full torso, i.e. dresses, jackets and coats. There is also the question of how current practice in clothing and pattern development informs and can control the placement of the sideseam in a manner applicable to the skills and understanding as well as the application of the user.

\subsection{Measurement divisions from TC2 software}

Table. 2. Arc divisions from TC2 output

\begin{tabular}{|c|c|c|c|c|c|c|}
\hline & 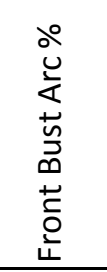 & 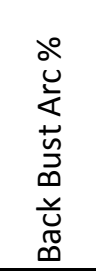 & 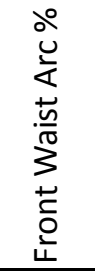 & 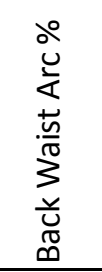 & 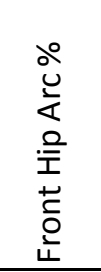 & 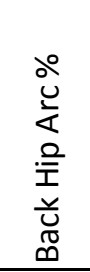 \\
\hline AsianAlva & 54.02 & 45.98 & 50.27 & 49.73 & 48.75 & 51.25 \\
\hline DressRite_size 8 & 54.57 & 45.43 & 49.96 & 50.04 & 48.22 & 51.78 \\
\hline MMU-WMS012C-0807 & 54.14 & 45.86 & 49.83 & 50.17 & 48.95 & 51.05 \\
\hline MMU-WMS012S-0807 & 54.26 & 45.74 & 49.95 & 50.05 & 48.13 & 51.87 \\
\hline WMS0006-0507 & 54.96 & 45.04 & 50.30 & 49.70 & 48.42 & 51.58 \\
\hline Wolf_m1959_size14 & 53.03 & 46.97 & 49.93 & 50.07 & 48.15 & 51.85 \\
\hline Wolf1997_size3 & 51.10 & 48.90 & 50.14 & 49.86 & 47.63 & 52.37 \\
\hline Average & 53.73 & 46.27 & 50.05 & 49.95 & 48.32 & 51.68 \\
\hline
\end{tabular}

The arcs defined by the TC2 software provide measurements that show the proposed proportions of the body in the back and front of full circumferences. Whilst the waist shows a near equal division in the population chosen, possibly due to the limited nature of dress forms in reflecting current bodies, the bust suggests as would be expected a larger portion to the front, clearly influenced by projection of the bust. Similarly, the hips show a larger projection to the back arc, which would be relative to the projection of the buttocks. These arcs however do not address the idea of balance within the garment or consider in their division the control areas of the body from which garments hang.

\subsection{Analysis of bodice patterns}

Analysis of bodice patterns constructed using nine different methods of flat drafting systems provided the opportunity to establish relative arc divisions that occurred during the draft process. The following (Table 1) shows the variation in arc percentages within these drafts when constructed for a UK size 12 dress form, as the methods of determining arcs is not explicit it is currently unclear which methods would always provide stable divisions and which would change relative to different measurements of the dress form. 
Table. 3. Arcs determined in current bodice construction methods

\begin{tabular}{|c|c|c|c|c|}
\hline & Fr Waist Arc & Bk Waist Arc & Fr Bust arc & Bk Bust arc \\
\hline Total girth on dress form & \multicolumn{2}{|c|}{70.2} & \multicolumn{2}{|c|}{90.6} \\
\hline Armstrong 2006 & 50.72 & 49.28 & 55.53 & 44.47 \\
\hline Aldrich 2004 & 50.13 & 49.87 & 52.75 & 47.25 \\
\hline Bray 2003 & 56.20 & 43.80 & 54.41 & 45.59 \\
\hline Beazley and Bond 2003 & 53.33 & 46.67 & 52.52 & 47.48 \\
\hline Shoben and Ward 1987 & 48.70 & 51.30 & 50.20 & 49.80 \\
\hline Bunka 2008 & 57.67 & 42.33 & 54.04 & 45.96 \\
\hline Haggar 2004 & 48.79 & 51.21 & 50.00 & 50.00 \\
\hline Kunick 1984 & 54.95 & 45.05 & 53.11 & 46.89 \\
\hline Campbell 1980 & 50.68 & 49.32 & 53.29 & 46.71 \\
\hline Average & 52.35 & 47.65 & 52.87 & 47.13 \\
\hline Min & 48.70 & 42.33 & 50.00 & 44.47 \\
\hline $\operatorname{Max}$ & 57.67 & 51.30 & 55.53 & 50.00 \\
\hline
\end{tabular}

\section{Conclusions}

Current practice indicates that there needs to be a more evolved approach to determining arc division within body scanning systems and the facility to define sideseams driven by garment considerations is of crucial importance. Current pattern construction methods show a variation in proposed arc proportions and in many cases, these are very different from the arcs defined automatically in TC2 scan software. Further analysis is required to tie together the varied methods of arc division according to practitioners, pattern construction methods and body scanning technology.

It is however clear that some methods of automated sideseam placement, determined by arc divisions, will not always define a vertical line that is consistent above and below the waist. Further integration of measurement application in pattern construction is required to drive developments in scanning technology to define a sideseam, which meets practitioner expectations and is understandable by those using data in the clothing industry. A clear contribution to this field would be determination of how a sideseam division should occur on the body and what it should be relative to in a virtual environment that can drive automation of pattern construction and help evolve practice in a manner readily understood by current and future practitioners.

\section{References}

1. Gill, S. and N. Chadwick, Determination of ease allowances included in pattern construction methods. International Journal of Fashion Design, Technology and Education, 2009. 2(1): p. $23-31$.

2. $\quad$ Aldrich, W., Chapter 1:History of sizing systems and ready-to-wear garments, in Sizing in Clothing, S. Ashdown, Editor 2007, Woodhead Publishing: Cambridge. p. 1-56.

3. Silberberg, L. and M.M. Shoben, The Art of Dress Modelling1998, London: LCFS Fashion Media.

4. Bray, N., Dress Pattern Designing: the basic principals of cut and fit: Classic Edition. 5th ed2003, Oxford: Blackwell. 
5. Kang, T.J. and S.M. Kim, Development of three-dimensional apparel CAD system, Part 1:flat garment pattern drafting system. International Journal of Clothing Science and Technology, 2000. 12(1): p. 26-38.

6. Kang, T.J. and S.M. Kim, Development of three dimensional apparel CAD system, Part 2: prediction of garment drape shape. International Journal of Clothing Science and Technology, 2000. 12(1): p. 39-49.

7. Tao, X. and P. Bruniaux, toward advanced three-dimensional modeling of garment prototype from draping technique. International Journal of Clothing Science and Technology, 2013. 25(4): p. 266-283.

8. Hlaing, E., C., S. Krzywinski, and H. Roedel, Garment prototyping based on scalable virtual female bodies. International Journal of Clothing Science and Technology, 2013. 25(3): p. 184197.

9. $\quad$ Armstrong, H.J., Pattern Making for Fashion Design. 5th ed2010, Upper Saddle River, N.J.: Pearson/Prentice Hall.

10. Aldrich, W., Metric pattern cutting for Women's wear. 5th ed2008, Oxford: Blackwell.

11. Bunka Fashion, C., Fundamentals of Garment Design2009, Tokyo, Japan: Bunka Publishing Bureau.

12. Beazley, A. and T. Bond, Computer-aided pattern design and product development2003, Oxford: Blackwell Science.

13. Gill, S., Determination of ease allowances included in pattern construction methods in Textile Institute 100th World Conference2010: Manchester, UK.

14. Kang, T.J. and S.M. Kim, Optimized garment pattern generation based on three-dimensional anthropometric measurement. International Journal of Clothing Science and Technology, 2000. 12(4): p. 240-254.

15. Farmer, B.M. and L.M. Gotwals, Concepts of Fit: An Individualized Approach to Pattern Design1982, New York, NY: Macmillan.

16. Rasband, J.A., Fabulous Fit1994, New York, NY: Fairchild Publications.

17. Hazen, G.G., Fantastic Fit for Every Body1998, Emmanaus, PA: Rodale Press.

18. Liechty, E.G., D.N. Pottberg, and J.A. Rasband, Fitting \& Pattern Alteration: A Multi-method Approach1992, New York, NY: Fairchild Fashion and Merchandising Group.

19. Lenker, S., Vogue Fitting: The Book of Fitting Techniques, Adjustments, and Alterations1987, New York, NY: Harper \& Row.

20. Ashdown, S., S. Choi, and E. Milke, Automated side-seam placment from 3D body scan data. International Journal of Clothing Science and Technology, 2008. 20(4): p. 199-213.

21. Aldrich, W., pattern cutting for women's tailored jackets: classic and contemporary2001: Blackwell science. 120.

22. Erwin, M.D., L.A. Kinchen, and K.A. Peters, Clothing for Moderns. 6th ed1979, New york: Macmillan Publishing.

23. ISO 7250-1:2010, Basic human body measurements for technological design - Part 1: Body measurement definitions and landmarks, 2010, International Standards Organisation: Brussels. 